\title{
Higgs Quantum Space Dynamics and the Nature of the Gravitational Fields
}

\author{
Jacob Schaf \\ Universidade Federal do Rio Grande do Sul (UFRGS), Instituto de Fsica, Porto Alegre-RS, Brazil \\ Email: schaf@if.ufrgs.br
}

How to cite this paper: Schaf, J. (2018) Higgs Quantum Space Dynamics and the Nature of the Gravitational Fields. Journal of Modern Physics, 9, 395-418. https://doi.org/10.4236/jmp.2018.93028

Received: January 9, 2018

Accepted: February 5, 2018

Published: February 8, 2018

Copyright (C) 2018 by author and Scientific Research Publishing Inc. This work is licensed under the Creative Commons Attribution International License (CC BY 4.0).

http://creativecommons.org/licenses/by/4.0/

\begin{abstract}
This work discusses the implications of the Higgs theory on Einstein's General Relativity, showing that these implications accurately lead to all the experimental observations. The Higgs theory introduces the Higgs field breaking the electroweak symmetry and filling up the universe with the Higgs quantum condensate. This Higgs Quantum Space (HQS) gives inertial mass to elementary particles by the Higgs mechanism and hence necessarily is the local ultimate reference for rest and for motions. Velocity with respect to the local HQS and not relative velocity is the origin of all the effects of motion on matter-energy. Within this scenario, the observed absence of the gravitational slowing of the GPS clocks by the solar field, as well as the absence of light anisotropy with respect to earth, demonstrates that earth and also the other planets of the solar system are very nearly stationary with respect to the local HQS. This can make a sense only if the HQS is itself moving round the sun according to a Keplerian velocity field, consistent with the planetary motions. The current theories explain the absence of the solar gravitational slowing of the GPS clocks with base in the principle of equivalence. Accordingly, the local Lorentz frame (LF), moving with earth round the sun, cancels the spacetime curvature and reproduces locally the environment of special relativity. In this local environment, earth and the GPS clocks are stationary and therefore display proper time. In the scenario of the present HQS dynamics, the local HQS materializes the local LFs, turning them into local proper LFs, stationary with respect to the local HQS. In its motion, the HQS carries these local proper LFs with it round the sun. The orbiting earth is stationary with respect to the local moving HQS and necessarily too with respect to these local proper LFs, thereby canceling the effects of the gravitational field and perfectly implementing the principle of equivalence. The present work shows moreover that this Keplerian velocity field of the HQS accurately creates the observed gravitational dynamics and all the effects of the gravitational fields on matter, on light and on clocks.
\end{abstract}




\section{Keywords}

Gravitation, Gravitational Fields, Theory of Relativity, General Theory of Relativity, Relativistic Effects, Gravitational Time Dilation, Higgs Theory, Relativistic Experiments

\section{Introduction}

According to Einstein's Special Theory of Relativity (STR), empty space (vacuum), by it alone, is unable to produce any physical effect [1] [2]. Only relative motions between observers are relevant in physics. The Principle of Relativity and the intrinsic constancy and isotropy of the velocity of light are the land-marks upon which the STR was constructed. In the construction of the General Theory of Relativity (GR), which is Einstein's theory of gravitation, the fundamental assumption is the principle of the equivalence of gravitational and inertial effects.

Gravity however is clearly an inertial dynamics created by empty space (vacuum). This demonstrates that empty space is much more than simply emptiness. If the orbital motion of earth cancels locally the effects of the gravitational field and recovers the condition of a stationary laboratory in a local special relativistic environment, as recently demonstrated by the GPS clocks, then the quintessence of the gravitational field necessarily is a velocity field of empty space itself, consistent with the orbital motion of earth. In the coming sections of this work, it will be shown that the Higgs Quantum Space, giving inertial mass to the elementary particles by the Higgs mechanism, moving round the sun according to a Keplerian velocity field, consistent with the orbital velocities of the planets, accurately creates all the observed effects of the gravitational fields on matter, on light and on clocks.

In GR, Einstein imputes the gravitational dynamics to curvature of the four-dimensional spacetime [1] [2]. In the neighborhood of a gravitational source, this curved spacetime is characterized by the invariant length of the four-dimensional line element $\mathrm{d} s$ that, for sufficiently weak gravitational fields, has the form:

$$
\mathrm{d} s^{2} \approx\left[1-\frac{2 U}{c^{2}}\right]^{-1} \mathrm{~d} r^{2}+r^{2} \mathrm{~d} w^{2}-c^{2}\left[1-\frac{2 U}{c^{2}}\right] \mathrm{d} t^{2}
$$

The coefficients $\left(1-\frac{2 U}{c^{2}}\right)^{-1}$ and $-c^{2}\left(1-2 U / c^{2}\right)$ are respectively the diagonal $g_{11}$ and $g_{44}$ components of the Schwarzschild metric tensor. The last term of this equation expresses gravitational time dilation, where $\mathrm{d} t$ is a time interval (measured by a clock, stationary in the gravitational field), $\mathrm{d} r$ is the measured radial distance within the gravitational field and $2 U=2 G M / r$ is the square of the local escape velocity from the gravitational field. The last term 
shows that, within the gravitational field, the rate of clocks is slowed. This means that the oscillation period $T(r)$ of the time standard, by which a clock counts time, is longer and that the rate of the time evolution of all physical processes is lower. The period of the time standard $T(r)$, by which the clocks count time, varies according to:

$$
T(r)=T_{0}\left(1-2 U / c^{2}\right)^{-1 / 2}
$$

where $T_{0}$ is the time period for $(U=0)$.

By the curved spacetime, GR can predict the gravitational dynamics in the solar system and also predicts many observed effects of the gravitational fields on light and on clocks. However, while the atomic clocks, stationary within a gravitational field, show exactly the gravitational slowing predicted by GR, recent experimental observations, achieved with the help of the GPS clocks, moving with earth round the sun, show that the gravitational slowing by the solar field is absent on them. This observation demonstrates that the orbital motion of earth cancels locally the effects of the gravitational field. Current theories explain this observation in terms of the principle of equivalence and motion of the local Lorentz frame (LF) together with earth. Accordingly, the orbital motion of earth cancels the effects of the gravitational field on matter, on light and on the clocks, recovering, in the local region of the moving earth, the environment of the STR, in which earth is stationary.

The Higgs theory introduces the Higgs quantum condensate (HC) or Higgs Quantum Space (HQS). By the Higgs mechanism, the HQS provides the physical origin of the inertial mass and the inertial behavior of the elementary particles. According to the Glashow-Weinberg-Salam electroweak model, the HQS is a very powerful spatial medium confining and localizing the elementary particles to within $10^{-19} \mathrm{~m}$. The HQS thus necessarily is the local ultimate reference for rest and for motion of matter-energy. It materializes at each point of space a local ultimate reference for rest and for motion. The HQS and these local references however can themselves be moving according to a well defined velocity field. Motion of this HQS, according to a non-uniform velocity field, creates inertial dynamics, which, after Einstein's equivalence of gravitational and inertial effects, is gravitational dynamics. Thereby, HQS dynamics directly implements the principle of equivalence. The HQS materializes the local Lorentz frames $(L F s)$, turning them into proper Lorentz frames, stationary with respect to the local HQS and carries them with it in its motions. Only the local LFs, stationary with respect to the local HQS, are proper LFs. The local HQS and the local proper LFs represent the local reference for absolute rest and absolute motion. On the other hand, motion with respect to the local HQS and the local LFs is the true origin of all the effects of motion on matter, on light and on clocks, the so called relativistic effects.

In the coming Sections of this work, it is shown that the absence of the solar gravitational slowing of the GPS clocks as well as the absence of the light aniso- 
tropy with respect to earth is the authentic signature of the physical mechanism of gravity in action. The absence of these effects, observed on earth, demonstrates that the HQS and the local LFs are moving round the sun according to a Keplerian velocity field, consistent with the planetary orbital motions. On moving round the sun, the HQS carries with it the local proper LFs. In the local region of the HQS and within the local proper LF of earth the solar gravitational pull is null, the velocity of light is isotropic with respect to earth and the clocks, moving with earth, display proper time. This immediately implements the conclusions of the current theories. This Keplerian velocity field of the HQS, carrying with it the local proper LFs is the quintessence of the gravitational fields.

The next Section 2 briefly describes the properties of the quantum condensates and highlights their role in the origin of the inertial dynamics of matter-energy, observed within the gravitational fields. Section 3 outlines the HQS dynamics, giving rise to the observed gravitational dynamics. Sections 4 and 5 make a rigorous mathematical analysis of the gravitational dynamics within the solar system and on earth. Details about the numerous predictions of the HQS dynamics gravitational fields on matter, on light and on clocks, all matching the experimental observations, can be found in Refs. [3] [4] [5] [6].

\section{The Higgs Theory and the Origin of the Inertial Mass}

Einstein has tried to explain the origin of the inertial mass of matter bodies in terms of Mach's ideas. Accordingly, the inertial mass of a local matter body arises from its connection with the other masses in the universe. This however entails instantaneous action from the infinite, which opens more problems than it solves.

Only fifty years later, has the Higgs theory provided a sane physical explanation for the origin of the inertial mass of the elementary particles. Accordingly, the inertial mass of the elementary particles, porting hypercharges, arises, not from coupling to the infinite, however from coupling of the particle fields to the local Higgs quantum condensate (HC), a spatial quantum fluid medium analogous to the superconducting condensate (SCC).

Quantum condensation of bosons is caused by the Bose-Einstein (BE) phase correlation between the boson wave-functions. This correlation leads to spontaneous breakdown of the $U(1)$ gauge symmetry of the boson system, resulting in condensation into a phase coherent macroscopic quantum state that can be described by a complex macroscopic Ginsburg-Landau order parameter $\Psi(r, \theta)=\psi(r) \mathrm{e}^{\mathrm{i} \theta}$, where $\psi(r)$ is an amplitude and $\mathrm{e}^{\mathrm{i} \theta}$ is a phase factor [7]. The ground state of the condensate is characterized by a well-defined phase $\theta_{0}$ that is arbitrarily selected between zero and $2 \pi$. The fact that this phase can take values between zero and $2 \pi$, without changing the energy of the condensate, proves that the gauge symmetry of the Lagrangian is preserved in the condensation. In the condensate, the principle of uncertainty for the bosons becomes singular. The uncertainty in momentum tends to zero and the uncertainty in po- 
sition diverges; the uncertainty in energy tends to zero and the uncertainty in time diverges. The condensate moreover is perfectly conservative. All steady excitations cost a well-defined quantized amount of energy and are indefinitely persistent. For instance, the super-current in a charged superconducting magnet in its persistent mode flows forever. It can be stopped only by an opposite electromotive force.

The $\mathrm{BE}$ phase correlation gives rise to a negative potential energy (bonding) term, the value of which increases linearly with the condensate density $\rho=\Phi^{*} \Phi$. Another positive potential energy (anti-bonding) term arises from repulsive core interaction between the bosons, that increases with the squared density $\rho^{2}$ and prevents collapse of the boson system. The effective potential $U(\rho)$ is the sum of these two terms that has the form:

$$
U(\rho)=-n\left(\Phi^{*} \Phi\right)+m\left(\Phi^{*} \Phi\right)^{2}
$$

where the value of the negative coefficient $(-n)$ of the bonding term is considerably larger than the positive coefficient $(+m)$ of the anti-bonding term. Therefore the minimum of the effective potential energy occurs, not for $\rho=0$, however for a finite value $\Phi^{*} \Phi=n / 2 m$, which is known as a non-zero vacuum expectation value. This effective potential is a local parameter that is highly homogeneous throughout the volume of the condensate.

The Higgs theory [8] [9] [10] introduces a scalar field, present throughout space, breaking the electro-weak symmetry and also spontaneously causes breakdown of the $U(1)$ symmetry of the weak force doublet, however letting the $U(1)$ symmetry of the electromagnetic field unbroken. Breakdown of the $U$ (1) symmetry at $10^{15} \mathrm{~K}$ gives rise to the Higgs condensate (HC) filling up the whole of space. The HC is the quintessence of the empty space (quantum vacuum), to be named Higgs Quantum Space (HQS). The nature of the HC or HQS, although formed by chargeless and spin zero bosons is totally analogous to the SCC and too can be described by a complex two component macroscopic Ginsburg-Landau order parameter [7]. It however is stabilized by a huge energy gap that, according to the Glashow-Weinberg-Salam electroweak model, achieves $200 \mathrm{GeV}$ [11] [12]. This energy gap is $10^{14}$ times larger than the energy gap of about $1 \mathrm{meV}$ of the usual quantum fluids (superfluids and superconducting condensates).

According to the gauge theories, all the elementary particles should originally be mass-less and be moving at the velocity of light. The first clue, that coupling of a field to a quantum condensate leads to confinement (reduction of the uncertainty in position) of the field and mass terms of the confined field (increase of the uncertainty of momentum and consequently of the inertial mass), was discovered by Anderson in superconductivity [13]. Gauge transformations of the macroscopic Ginsburg-Landau superconducting order parameter $\left(\Psi(r, \theta)=\psi(r) \mathrm{e}^{i \theta}\right)$, in the presence of a magnetic field, confined by the Meissner effect [14], reveal mass terms. The vector potential of the magnetic field causes phase perturba- 
tions of the superconducting (SC) order parameter, elevating the energy of the superconducting condensate (SCC). Confinement of the magnetic field by the Meissner effect reduces this energy. The gauge transformations obviously do not create mass. They only reveal it on testing the mobility of the condensate in the presence of the confined field. The reason is that gauge transformations involve local additional phase perturbation of the superconducting order parameter that entails accelerations of the SCC (creation of additional screening currents) and increase of its momentum and energy. This is the physical mechanism generating inertial mass of the photons within superconductors.

The inertial mass of elementary particles, porting hypercharges, is generated by the Higgs mechanism, that is totally analogous to the well-known Meissner effect in superconductivity. The Higgs mechanism [8] [9] [10] is the perfect HQS analog of the Meissner effect in superconductivity. It confines the weak field of the vector bosons $(\mathrm{W}+, \mathrm{W}-, \mathrm{Z})$ of the weak nuclear force field to within $10^{-19} \mathrm{~m}$ and also confines quarks and leptons via a Yukawa like mechanism, thereby giving inertial mass to these elementary particles. Likewise the Meissner effect confines the electromagnetic field within the superconductor, creating inertial mass for the photons, the HC or HQS confines the weak and strong nuclear fields by a direct or indirect Higgs mechanism, creating inertial mass for the elementary particles within the empty space (vacuum).

Quantum fluids, by their very nature, are well-known to be extremely subtle and practically inaccessible to our direct perception. However, the role of the Higgs mechanism in the universe is absolutely crucial. Without the Higgs mechanism, the existence of our universe simply would be impossible. The macroscopic quantum physics of the HQS, enforcing localization and creating inertial mass for the elementary particles by the Higgs mechanism, necessarily rules their inertial dynamics and is their ultimate reference for rest and for motion. The HQS materializes a local ultimate reference for rest and for motion, a local proper Lorentz frame (LF), stationary with respect to the local HQS. In its motion, the HQS carries the local proper LFs with it. Velocity with respect to the local HQS and not relative velocity is the origin of all the effects of motion. The HQS plays a fundamental role in the physics of the microscopic quantum world as well as in the physics of the macroscopic world of gravitation. On creating mass, the HQS too is responsible for the gravitational fields, because it is mass that creates the gravitational fields. The HQS thus is the obvious link between quantum physics and gravitation [3] [4] [5] [6].

The Higgs theory introduces fundamental changes in Einstein's view about the nature of empty space (vacuum) and about the meaning of motions. The HQS is much more than simply a local reference for rest and for motions. It literally governs the inertial motion of matter-energy and thus necessarily is the local ultimate reference for rest and for motions. Several recent experimental observations [15] [16] demonstrate that the velocity of light is isotropic and constant (fixed) with respect to the local HQS and not with respect to every 
possible inertial reference [3] [4] [5] [6]. In fact the constancy of the velocity of light, measured by the usual method of light go-return round-trips and clock, is an experimental artifact. It is an experimental artifact because, in this method, the velocity of light is the ratio between the round-trip time of light between two mirrors and the round-trip time of the electromagnetic field in the time standard (Cs atoms), by which the atomic clocks count time. These two round-trip times are both affected by motion with respect to the local HQS in exactly the same proportion, as demonstrated by several experiments, like, for instance, the Ives-Stilwell type of experiments [17].

In the scenario of the HQS all effects of motion are due exclusively to velocity with respect to the local real HQS and not to relative velocity. This breaks the reciprocal symmetry of the theory of relativity (TR) that is one of the stalemates of the TR and the source of numerous unsolvable paradoxes. The real HQS is the local quantum medium, ruling the motion of matter-energy. This has the consequence that the velocities of particles and the true velocity of light, in empty space, have well-defined values with respect to the local HQS and not with respect to the reference of the observer. The velocity of light with respect to the local HQS is the limiting (maximum) propagation velocity of matter-energy and of any perturbation in the HQS. These facts establish a resemblance between the propagation of the matter-waves in the HQS and of the sound waves in the atmosphere. It is motion of the laboratory with respect to the local HQS that causes light anisotropy and consequently time dilation and slowing of all physical processes. It does so because anisotropic velocity of light and of electromagnetic perturbations in general, is well-known to cause increase of the round-trip time of light between two mirrors as well as an exactly proportional increase of the period of the time standards [17] by which the clocks count time, which slows the clock rates. The observed absence of the gravitational slowing of the GPS clocks by the solar field [15] [16] and the observed absence of light anisotropy with respect to earth [18] both consistently demonstrate that the planet earth is stationary with respect to the local HQS in the velocity field of the $H Q S$, creating the gravitational dynamics within the solar system. Both these observations are the signature of the same physical mechanism of gravity in action that is the subject in the next Section 3.

In the quantum world, all physical processes involve quantum transitions that entail the oscillations of the electromagnetic (EM) field perturbations, with emission or absorption of EM field quanta. As the propagation velocity of the electromagnetic perturbations in these fields is fixed with respect to the local HQS, the oscillation period of atoms increases with the velocity of the laboratory or of atoms [17] with respect to the local HQS and hence decreasing the rate of all physical, chemical and biological processes. The observed frequency shifts for dipole radiation however are different for transverse and longitudinal electromagnetic field oscillations (round-trips). While radiation along the longitudinal direction [17] is selective for dipole radiation emitted by transverse EM oscilla- 
tions in the sources, transverse directions are selective for longitudinal electromagnetic oscillations (with lower frequency). Moreover, relative velocity between light sources and observers leads to the well-known Doppler shifts.

The Higgs condensate (HC), likewise the SCC, too can locally move under phase gradients of the order parameter $\left.\Psi(r, \theta)=\psi(r) \mathrm{e}^{\mathrm{i} \theta}\right)$. As the velocity of light and of particles is well-defined with respect to the local HQS, in its motion, the HQS drags the electromagnetic waves and the de Broglie matter-waves. Non-uniform motion of the HQS causes non-uniform drag and thus a refraction rate of the matter-wave fronts, analogously as wind gradients refract sound waves. This refraction rate leads to inertial dynamics of matter-energy, which after Einstein's principle of equivalence, is gravitational dynamics [3] [4] [5] [6]. On one hand, this turns the origin of the gravitational dynamics conceptually simple, however, on the other hand, in order to describe the motion of matter-energy within gravitational fields, it is necessary to know precisely the velocity field of the HQS. This is the subject of the next Section 3.

Likewise the Meissner effect develops a velocity field of the SCC (screening currents) generating a macroscopic Lorentz force field, confining, compressing or expelling the magnetic flux out from a superconductor, the Higgs mechanism develops a velocity field of the HQS, generating a gravitational force field, macroscopically confining and compressing the matter fields. While the Lorentz force field is a macroscopic manifestation of the Meissner effect, the gravitational force field is a macroscopic manifestation of the Higgs mechanism. Both condensates thereby lower their energy.

\section{HQS Dynamics as the Quintessence of the Gravitational Fields}

According to General Relativity (GR), in a spherically symmetric gravitational field, all the inertial references are free falling along the radial coordinate with velocity equal to the local escape velocity. According to Einstein's principle of equivalence [1] [2], a free-falling elevator is an inertial reference (IR). However, it is well-known that, in a gravitational field, each local IR is valid at only one geometrical point and that any two IRs, not located exactly at the same point, are mutually non-inertial. Hence, Einstein's free-falling elevator cannot be a well-defined IR in space and also not along time. It also cannot be a proper Lorentz frame (LF). It in fact is a different IR at each point of space along its path. The fact that, in the view of an observer in the free-falling elevator, everything in the elevator seems like in a true IR is an experimental artifact, because it compares the free-falling observer with the free-falling elevator that are both at the same place and affected by gravity in exactly the same way. It is an experimental artifact, analogous to the measurement of the velocity of light by the method of light round-trips between two mirrors and a clock, which too are affected by motion in exactly the same proportion. Only full understanding of the true physical mechanism of gravity can elucidate this puzzle. 
In order to settle the riddle of the free-falling elevator, Einstein has conceived the principle of equivalence, the equivalence of the gravitational pull and the inertial pull. In the case of the freely falling elevator, the gravitational pull is locally canceled. With base in this observation, Einstein has concluded that a free-falling clock or a clock moving in a circular orbit, the effects of the gravitational field are locally canceled and that the clock must display proper time. Moreover, in order to explain the fact that, in the locally canceled gravitational field, earth and the local Lorentz frame as well as the GPS clocks go on moving along a circular path (curve), in the absence of any centripetal force, Einstein has introduced the idea of the curved spacetime and the generalized inertial motion along the geodesics in this curved spacetime. This spacetime curvature is characterized by the invariant length of the four-dimensional line element, specified by Equation (1) in Section 1. The absence of the solar gravitational slowing of the GPS clocks, moving with earth round the sun, usually is seen as the experimental confirmation of the principle of equivalence.

Clocks stationary within a gravitational field display exactly the gravitational slowing predicted by Equations (1) and (2). Usually, this slowing of the stationary clocks is imputed to the implicit upward acceleration $g(r) \boldsymbol{e}_{r}$, stopping their free-fall within the gravitational field. However, accelerations of Muons in cyclotrons, up to $10^{19} \mathrm{~m} / \mathrm{sec}^{2}$, demonstrate that accelerations do not at all increase their mean lifetimes [19]. The increase of the observed lifetime is due exclusively to their (linear) velocity. This observational fact definitively invalidates the allegation that the implicit upward acceleration of clocks, stationary in the gravitational field, is the cause of the gravitational slowing. Unfortunately, actually there are no direct experimental observations that can falsify the allegations that a free-falling clock displays or not proper time.

Einstein's model of the free-falling inertial references (IRs) has another shortcoming. It cannot produce the gravitational pull on matter, observed on earth. It cannot because at each fixed point $r_{0}$ of space, the free-fall velocity $v\left(r_{0}\right)$ of the local IRs is fixed, equal to the local escape velocity $\left(2 G M / r_{0}\right)^{1 / 2}$ from the earth's field. At each position $r_{0}$ the change of the free-fall velocity of the free-falling IRs is zero $\left(\mathrm{d} v /\left.\mathrm{d} t\right|_{r_{0}}=0\right)$. According to the principle of inertia, a particle or matter body brought to rest at any fixed position $r_{0}$ and then abandoned, will remain in equilibrium there. Any perturbation however will initiate a runaway departure, upward or downward. In order to effectively initiate a free-fall or produce the observed gravitational pull, a locally dynamic space is necessary, which, in Einstein's model, would require $\mathrm{d} v /\left.\mathrm{d} t\right|_{r_{0}}=\boldsymbol{g}$. However, in this case, the free-fall velocity of the local IRs rapidly would exceed the velocity of light, which is absurd.

The only way of a dynamic space to naturally generate the gravitational acceleration and the observed gravitational pull, without ending in a stalemate, is one based in variation of the direction of a local velocity vector $v$ of the particles. This creates a centrifugal acceleration, which must point from 
everywhere toward the gravitational center. This becomes possible only if a particle, initially stationary in a gravitational field, has a horizontal implicit velocity, equal and opposite to the local direct circular orbital velocity, and the direction of which is continuously rotated. Only a non-uniform velocity field of the local HQS can generate such an inertial dynamics. In the coming paragraphs, it will be shown that a Keplerian velocity field of the HQS, besides adequately generating the gravitational pull as a genuine centrifugal effect, also correctly creates the gravitational clock slowing, observed on earth, correctly leads to the absence of the gravitational slowing of the GPS clocks by the solar field and too leads to the absence of light anisotropy with respect to earth.

Einstein has related the gravitational slowing of clocks, stationary in the gravitational field, to the local escape velocity, which is the free-fall velocity of the local inertial references. However, the escape velocity is a local characteristic of the gravitational field that cannot depend on the orbital velocity of earth. In the case of the GPS clocks, if Einstein's approach, in Equation (2) were straightforwardly valid (without additionally appealing to the principle of equivalence and the local cancelation of the effects of the gravitational field), it would lead to time delays of about 24 ns during the 6 hours closer than earth from the sun, which would be recovered during the 6 hours farther from the sun. This delay is two decimal orders of magnitude larger than the precision of the clocks during the 12 hours of the orbital motion. However, the GPS clocks, moving with earth round the sun, have revealed that both the solar gravitational slowing as well as the special relativistic slowing, due to the orbital velocity round the sun simply are absent [15] [16]. Only the small gravitational slowing by the earth's field is observed. Einstein's original spacetime curvature cannot explain this absence.

Currently, the absence of the solar gravitational slowing of the GPS clocks is explained with base in the principle of equivalence, according to which, earth is stationary with respect to the local Lorentz frame (LF) [20]. It is alleged that in the local LF of the orbiting earth the GPS clocks are stationary and therefore are not slowed by the solar field and display proper time. It is assumed that, from the viewpoint of the local LF, the solar gravitational field and the local spacetime curvature are canceled, recovering the flatness of special relativity. It also is stated that the GPS clocks, orbiting with earth round the sun, are not slowed by the solar field, because they are free-falling with earth in the solar field. However, then strangely it is alleged that these same GPS clocks, moving in circular orbits round earth, are slowed by the earth field $\left(1.67 \times 10^{-10} \mathrm{sec} / \mathrm{sec}\right)$ because they are continuously changing their LF with respect to the ground observer. Relative motion with respect to the terrestrial observer could in principle give rise to Doppler shifts and special relativistic effects. However, from the viewpoint of the principle of equivalence, specifically the gravitational slowing should be absent. If the GPS clocks, in orbit round the sun, are free-falling with earth in the solar field, they of course too are free-falling with the GPS satellites in the earth's field, which should cancel the effects of the earth's gravitational field. Why in one case 
does the orbital motion cancel the gravitational slowing and in the other identical kinematical situation it does not cancel it?

Also strangely it is alleged that a clock in free-fall along a radial coordinate does not run slow, because this cancels the gravitational field in the local Lorentz frame. When however this clock is stopped, then it will run slow. This entails the conclusion that the implicit upward acceleration in the gravitational field is the cause of the clock slowing. This however is in frontal conflict with the experimental observations of Muons in cyclotrons that show that acceleration causes no time dilation [19]. In fact, the free-falling clocks should display, besides the gravitational slowing, the special relativistic slowing, due to free-fall velocity and the Doppler shift for observation by a non-free-falling observer. Clearly, the simple free-fall and the simply orbital motion cannot be the true reason for the absence of the gravitational slowing of the GPS clocks by the solar field. The explanation in terms of the principle of equivalence is a hypothetical Aristotelian like explanation. Einstein's GR can model and describe many effects of the gravitational fields with base in postulates and principles. It however, does not supply the real underlying physics, powering these principles. Only full understanding of the physics, implementing these principles, can elucidate the true hush-hush of the gravitational fields.

The Higgs theory introduces profound changes in Einstein's view about the nature of empty space (vacuum) and about the meaning of motions. The Higgs Quantum Space (HQS) gives mass to the elementary particles by the Higgs mechanism and therefore literally governs the inertial motion of matter-energy. The HQS necessarily is the local ultimate (local absolute) reference for rest and for motions. Motion of matter-energy with respect to the local HQS gets locally an absolute meaning and all effects of motion are due exclusively to the velocity with respect to the local real HQS and not to relative velocity. If this HQS locally moves in the ordinary space, it carries with it the local ultimate reference for rest and for motions. A particle or body, stationary with respect to the ordinary space, in the moving HQS, necessarily is implicitly moving with respect to the local HQS. This motion is real and gives rise to all the well-known effects of motion, light anisotropy, clock slowing, spectral red-shifts etc. In a reference moving with respect to the local HQS the velocity of light is anisotropic and a clock, stationary in this reference, runs slow. The HQS potentially can provide all the physics underlying Einstein's principles backing GR.

The HQS of the Higgs theory materializes the local Lorentz frames (LFs), turning them into real local proper LFs intrinsically stationary with respect to the local HQS. In its motion, the HQS carries these local proper LFs with it. Such local proper LFs are indissoluble from the local HQS. Only the local LFs, stationary with respect to this local HQS, are proper LFs. Any LF moving with respect to the local HQS and to the local proper LF is not a proper LF. Also, any motion of the reference with respect to the local HQS and thus, with respect to the local proper LFs or vice-versa, gives rise to light anisotropy and to slowing of 
the clock rate. However, non-uniform velocity of the local HQS and thus of the local proper LFs in the ordinary space gives rise to inertial dynamics, which, according to the principle of equivalence, is gravitational dynamics. The observed absence of the solar gravitational slowing on the GPS clocks and the absence of light anisotropy with respect to the orbiting earth, both consistently demonstrate that earth is stationary with respect to the local real HQS and also with respect to the local proper LF. This obviously can make a sense only if this HQS (the local ultimate reference for rest and for motion of matter-energy) is itself moving round the sun according to a velocity field, consistent with the orbital velocity of earth.

However, obviously earth cannot be in a privileged kinematical situation to the detriment of all the other planets and astronomical bodies in general throughout the universe. All the planets of the solar system must equally be very nearly stationary with respect to the local HQS and clocks moving with all these planets show very closely proper time. Hence, the macroscopic velocity field of the HQS, round the sun and creating the solar gravitational field must have the form of a Keplerian velocity field consistent with the planetary orbital motions:

$$
\boldsymbol{V}(r)=(G M / r)^{1 / 2} \boldsymbol{e}_{\phi}
$$

This Keplerian velocity field is a cylindrical velocity field, in which the magnitude of the velocity of the HQS along the $\phi$ coordinate is spherically symmetric, $G$ is the gravitational constant, $M$ is the mass of the gravitational source (sun), $r$ is the radial spherical coordinate and $\boldsymbol{e}_{\phi}$ is a unit vector along the azimuthal spherical coordinate $\phi$. Refs. [3] [4] [5] show that this Keplerian velocity field of the HQS accurately creates the observed gravitational dynamics and all the observed effects of the gravitational fields on light and on clocks. This Keplerian velocity field of the HQS is the quintessence of the gravitational fields. It provides the true origin of the inertial dynamics, observed within the gravitational fields, which, after Einstein's equivalence of gravitational and inertial effects, is gravitational dynamics.

As asserted previously, the HQS materializes the local LFs, turning them into local proper LFs and thereby implementing the principle of equivalence, turning the gravitational pull into a genuine inertial pull. It will turn out that the gravitational pull is a spherically symmetric field of centrifugal effects (fictitious forces) toward the gravitational center. In its circular equatorial orbital motion, earth follows very closely the motion of the local HQS and of the local proper LFs round the sun. Along this motion, Earth as well as the local proper LFs is strictly stationary with respect to the local moving HQS. In these conditions, the velocity of light is isotropic with respect to earth, not because of the intrinsic isotropy of light, however because earth is very closely stationary with respect to the local HQS. This is why, in the case of the orbital motion of earth, the approache of the HQS dynamics and the approach based in the principle of equivalence both make exactly the same predictions: They predict the local 
cancelation of the effects of the solar gravitational field, the absence of the solar gravitational slowing on the GPS clocks as well as the absence of light anisotropy with respect to earth. They also will straightforwardly give a genuine physical explanation to the fact that, in the case of the GPS clocks, moving round earth in non-equatorial circular orbits, making 55 degrees with respect to the earth's equator, are slowed by the earth field.

The Keplerian velocity field of the HQS implements, for the planets, the principle of equivalence, in the limit of zero gravitational effects and zero inertial pull. The cylindrical Keplerian velocity field of the HQS carries the local proper LFs with it along the $\phi$ spherical coordinate round the sun consistently with the planetary orbital velocities, according to Equation (4). This perfectly implements the usual allegation, with base in the principle of equivalence, that the local LFs, moving together with earth round the sun are proper LFs and that this is the reason why the rate of GPS clocks is not slowed by the solar field. In this Keplerian velocity field earth and the GPS clocks and also the local proper LFs, are stationary with respect to the local HQS. Neglecting the small effects of the earth's field on the GPS clocks, these clocks show very closely proper time.

The reason why the velocity of earth and of the astronomical bodies in general, throughout the universe, are so small with respect to the local HQS is intimately related with the wavelength stretching, caused by the expansion of the HQS together with the expansion of the matter universe. However, the averaging down of the momentum during the gravitational agglomeration of matter into large astronomical bodies too importantly contributes to this low velocity of the astronomical bodies with respect to the local HQS. The effect of the very small velocities of the planets (of only a few hundreds of meters per second) only gives rise to their slightly elliptic orbits.

According to the present HQS dynamics, clocks moving round earth along direct circular equatorial orbits too are stationary with respect to the local HQS and with respect to the local LFs and too are expected to display proper time. However, any clock, moving with respect to the local HQS and or with respect to the local proper $L F$, cannot display proper time. Clocks in a polar orbit round earth are expected to be slowed two times more than clocks stationary at the same altitude and clocks in retrograde circular equatorial orbits are slowed four times more than a stationary clock at the same altitude. In particular the GPS clocks moving round earth, along closely circular orbits, however making a $=55$ degrees with respect to the earth's equator have a considerable velocity with respect to the local HQS. They have a velocity component $3.87(1-\cos \alpha) \mathrm{km} / \mathrm{sec}$ with respect to the local HQS toward the West and also a North-South velocity component of $3.87 \sin \alpha \mathrm{km} / \mathrm{sec}$. The resultant velocity with respect to the local HQS is given by $3.87[2(1-\cos \alpha)]^{1 / 2}$, which gives $3.574 \mathrm{~km} / \mathrm{sec}$ at the largest part of the orbit. The average velocity along the whole orbit is approximately 3 $\mathrm{km} / \mathrm{sec}$. Analogously the Westward velocity of the earth-based station at 
Colorado highs with respect to the local HQS is about $7.5 \mathrm{~km} / \mathrm{sec}$. To these velocities of the satellites and of the ground based clock the velocity of the Cs atoms of $0.255 \mathrm{~km} / \mathrm{sec}$ within the atomic clocks must be added. Also the small transverse Doppler shift, due to the implicit velocity of the earth-based stations must be considered. Altogether these effects achieve closely the observed $4.5 \times 10^{-10} \mathrm{sec} / \mathrm{sec}$.

Corrections, due to non-spherical mass distribution as well as to perturbations by motions and rotations etc. are certainly important to get fine-tuning of the GPS. However, all these smaller effects together achieve not more than a few percents of the principal term of the gravitational clock slowing [20]. In the present work the cause of time dilation is unified. It has the same origin (velocity with respect to the local HQS) in free-space and within gravitational fields.

In the Keplerian velocity field of the HQS, the locally valid IRs are not free-falling as alleged in GR. Each local inertial reference (IR), valid at a given point of the ordinary space $\left(r_{0}, \theta_{0}, \phi_{0}\right)$, is rotating round an over-head axis, fixed in the ordinary space at the point $\left(2 r_{0}, \theta_{0}, \phi_{0}\right)$. The idea of rotating inertial references can seem to be confusion. It however is not at all, because here it is the HQS itself, the local ultimate reference for rest and for motion and governing the inertial motion of matter-energy that is so rotating. In order to each locally valid IR to be rotating round such a fixed over-head axis at $r^{\prime}=2 r$, each local IR, valid in a small region of space round $r$, needs to move together with the local HQS and the local proper LFs round the gravitational center according to the Keplerian velocity field, given by Equation (4). In fact the Keplerian velocity field of the HQS, specified by Equation (4) is the only possible one that is able to create this outside-inside and inside-outside centrifuge phenomenon, observed within the gravitational fields.

In this HQS dynamics, a body, stationary in the gravitational field, at the validity point of the local rotating inertial reference, is implicitly moving the whole time oppositely along a circular path round the same over-head axis as the local IR, under a Real upward centripetal force. Most importantly: As the velocity gradient of the Keplerian velocity field Equation (4) points, from all positions of space from the equator to the poles, toward the gravitational center, this velocity field has the remarkable virtue of generating a central spherically symmetric field of centrifugal accelerations (centrifugal forces) toward the gravitational center. This unconventional inertial dynamics, produced by the Keplerian velocity field of the HQS, is the true hush-hush of the gravitational fields that is so difficult to understand. It enforces us to the conclusion that gravitational effects are not simply equivalent to inertial effects, however are identically inertial effects.

The fact that, in the Keplerian velocity field (Equation (4)), the velocity of the HQS and of the local LFs increases with decreasing radial coordinate, has the consequence that the distribution of velocity, within any infinitesimal region in the $[r, \theta]$ plane, corresponds to rotation round the overhead axis. The local 
angular velocity is $W=\left((G M / r)^{1 / 2}\right) / r \boldsymbol{e}_{\theta} \mathrm{rad} / \mathrm{sec}$, where $\boldsymbol{e}_{\theta}$ is a unit vector along the $\theta$ spherical coordinate. This rotation is the active principle underlying the gravitational acceleration and the gravitational pull. However, in fact the Keplerian velocity field of the HQS and of the local proper LFs does not fully reproduce the local environment of the STR, because, in the Keplerian velocity field, the local inertial references are rotating round the overhead axis at an angular velocity $W=\left((G M / r)^{1 / 2}\right) / r \boldsymbol{e}_{\theta} \mathrm{rad} / \mathrm{sec}$, while in STR the local inertial references do not rotate at all. However, as the velocity of earth, with respect to the local HQS and the local LFs is very small, the effect of this rotation is only turning the orbit of earth a little bit elliptic.

The macroscopic velocity field Equation (4) of the HQS and of the LFs is created uniquely by the gravitational source. This is a macroscopic manifestation of the Higgs mechanism. By this Keplerian velocity field the HQS confines macroscopically the matter fields, thereby lowering the energy of the Higgs condensate. However, each particle or astronomical body, moving in the Keplerian velocity field, carries with it its own velocity field.

From the HQS dynamics viewpoint, the GPS clocks are of course not free-falling with earth in the solar field as assumed in GR. The GPS clocks simply are moving with earth and the local HQS and also with the local proper LFs round the sun in a direct nearly circular equatorial orbit and so are (very closely) stationary with respect to the local HQS and also with respect to the local proper LFs. This is the reason why they are not slowed by the gravitational field and why they display proper time. This also is the reason why the velocity of light is isotropic with respect to earth.

Only clocks moving together with the local HQS and with the concomitantly moving local proper LFs can display proper time. Only in a laboratory moving in a direct circular equatorial orbit, like earth round the sun and hence stationary with respect to the local HQS and also with respect to the local com-moving proper LFs, can the velocity of light be isotropic. This kinematical situation is not exceptional; however is the largely dominant situation of practically all the natural astronomical system throughout the universe. The natural astronomical systems throughout the universe (planetary satellite systems, solar systems, galaxies) are disk-shaped because this minimizes their velocity and their kinetic energy with respect to the local HQS. In Refs. [3] [4] [5] [6], it is shown that practically all the natural astronomical bodies throughout the universe are very closely stationary with respect to the local HQS and thus also with respect to the local proper LFs in the respective gravitational fields. This is the reason for the observed universality of the laws of physics. The reason for this universally slow velocity of the matter bodies is stretching of the wavelength $\lambda$ of the particles with the expansion of the HQS (universe) and the consequent slowing down of the momentum with respect to the local HQS, according to de Broglie's equation $(p=h / \lambda)$. However, the averaging down of 
the momentum during gravitational agglomeration into large astronomical bodies too plays an important role.

As the de Broglie matter wave of any particle necessarily extends over a finite region of space, the $\pm \phi$ velocity component of such a particle undergoes a local characteristic time rate of refraction (rotation of the velocity vector). A free particle or clock, stationary with respect to the ordinary space, within the gravitational field (Keplerian velocity field) is implicitly moving at a velocity $\boldsymbol{V}_{\text {impl }}(r)=-(G M / r)^{1 / 2} \boldsymbol{e}_{\phi}$ with respect to the local HQS and also with respect to the local proper LF. This velocity is implicit (Imaginary), because it cannot be described with respect to the ordinary space. Because of the velocity gradient of the Keplerian velocity field of the HQS, the $\pm \phi$ velocity component will rotate (refract) according to a locally well-defined time rate, at an angular velocity $\boldsymbol{W}=\left((G M / r)^{1 / 2}\right) / r \boldsymbol{e}_{\theta} \mathrm{rad} / \mathrm{sec}$. This rotation is the active principle of gravity that generates the gravitational acceleration. This implicit velocity also causes the gravitational clock slowing and light anisotropy. However, the effects of the Keplerian velocity field of the HQS of the earth field, in the sense of the Moon's orbital motion, on light and on clocks are very small $10^{-10}$ and hence very difficult to detect.

In fact, the rotation rate (refraction rate) is not the same for all the velocity components. It is not a trigonometric rotation. The $\pm r$ velocity component, for instance, does not see the motion and rotation of the local HQS through the $[r, \theta]$ plane that refracts the $\pm \phi$ velocity component. It however sees the opposite rotation rate of the HQS through the $[\theta, \phi]$ plane, due to the motion of the HQS round the gravitational center. The opposite rotation of the $\phi$ and the $r$ velocity components characterizes a hyperbolic rotation. In order to implement this hyperbolic rotation rate, the local HQS and the local proper LFs must move round the gravitational source along the $+\phi$ spherical coordinate $\left(\boldsymbol{e}_{\phi}\right)$ as given by Equation (4). In this Keplerian velocity field, the rotation rate $\boldsymbol{W}_{\phi}=+\left((G M / r)^{1 / 2}\right) / r \boldsymbol{e}_{\theta} \mathrm{rad} / \mathrm{sec}$ of the $-\phi$ velocity component and the $\boldsymbol{W}_{r}=-\left((G M / r)^{1 / 2}\right) / r \boldsymbol{e}_{\theta}$ rotation rate of the $-r$ velocity component rules the path (orbit) of the free particles in the gravitational field. The planets of the solar system are very closely stationary with respect to the local moving HQS and also with respect to the local LFs. The effect of the refraction of this very small velocity is quite irrelevant. It only makes the orbits slightly elliptic. The velocity of light with respect to the planets is very nearly $c$ and isotropic as effectively observed. Clocks moving with the planets, display very closely proper time.

Einstein could have concluded from the null light anisotropy results that earth is stationary with respect to the local space ruling the motion of matter and the propagation of light and that this space is moving round the sun, consistently with the Keplerian planetary motions. He also could have seen this Keplerian 
velocity field of space as the quintessence of the gravitational fields and incorporated it into his four-dimensional spacetime. He also could have concluded that this space is moving round the galactic center, consistently with the solar system and that it is expanding, consistently with the recession of the galaxies. Ref. [3] outlines such a universe and shows that it matches with incredible details all the observations without additional or bizarre hypotheses, like dark matter and dark energy. However, instead of this, Einstein has postulated the intrinsic isotropy and constancy of the velocity of light. This option has encumbered the simple implementation of the principle of equivalence to explain gravity and has sown the incompatibility between quantum physics and GR.

\section{The Gravitational Dynamics in the Solar System and on Earth}

The Keplerian velocity field of the HQS (Equation (4)), creating the solar gravitational field achieves $436 \mathrm{~km} / \mathrm{sec}$ on the solar surface. It simply carries the planets with it along direct circular equatorial orbits, while they are very closely stationary with respect to the local moving HQS and also with respect to the local proper LFs. This is their local resting condition with respect to the local $H Q S$. The orbital velocity cancels locally the effects of the gravitational field on matter, on light and on clocks. Light is closely isotropic with respect to earth and with respect to the other planets and clocks, moving with them, display proper time. By the fact that the velocity of earth with respect to the local HQS is only of a few hundreds of meters per second, the effect of the refraction rate of this small velocity is very low. It only makes its orbit a little bit elliptic. Please see Refs. [3] and [4] for the full details.

The velocity of the HQS in the Keplerian velocity field (Equation (4)) round earth, in the sense of the Moon's orbital motion and creating the earth's gravitational field is much smaller than in the solar field (only about $7.91 \mathrm{~km} / \mathrm{sec}$ on surface). However, as earth rotates only very slowly $(465 \mathrm{~m} / \mathrm{sec}$ at the equator), the earth-based laboratories are moving at an implicit velocity toward the West at velocities, ranging from $7.445 \mathrm{~km} / \mathrm{sec}$ at the equator up to 7.91 $\mathrm{km} / \mathrm{sec}$ at the poles, which creates the observed gravitational effects on matter, on light and on clocks on earth. Although very small, these effects are much more relevant on earth than those of the solar field on the moving earth. Due to the local velocity distribution of the HQS in the earth's Keplerian velocity field, the $-v_{\phi}(t)$ velocity components of objects within an earth-based laboratory, pointing toward the West (de Broglie matter wave-fronts in the $[r, \theta]$ plane) are refracted at a rate (angular velocity) $+\left[G M / r^{3}\right]^{1 / 2} \boldsymbol{e}_{\theta}$ and the $-v_{r}(t)$ velocity component (wave-fronts in the $[\theta, \phi]$ plane) of this object will be refracted in the opposite sense, at a rate $-\frac{1}{2}\left[G M / r^{3}\right]^{1 / 2} \boldsymbol{e}_{\theta}$. The $v_{\theta}$ velocity component will not be refracted at all, because the Keplerian velocity field 
Equation (4) has no component along the $\theta$ coordinate. The refraction rates of the three components are given by:

$$
\begin{gathered}
\boldsymbol{W}_{r}(r)=-\frac{1}{2}\left[G M / r^{3}\right]^{1 / 2} \boldsymbol{e}_{\theta} \\
\boldsymbol{W}_{\phi}(r)=+\left[G M / r^{3}\right]^{1 / 2} \boldsymbol{e}_{\theta} \\
\boldsymbol{W}_{\theta}(r)=0
\end{gathered}
$$

Refs. [3] [4] [5] [6] explain in detail how to find these rotation rates. Equation (5) specify an asymmetrical hyperbolic rotation rate that accomplishes the Virial theorem, assures conservation of the angular momentum and of the total mechanical energy.

The refraction rate of the implicit velocity $-\boldsymbol{V}_{\text {impl }}(r)=-(G M / r)^{1 / 2} \boldsymbol{e}_{\phi}$ of an initially stationary particle, according to Equation (5b), generates a Real vertical downward acceleration given by:

$$
\boldsymbol{g}(r)=W_{\phi}(r) \boldsymbol{e}_{\theta} \times V_{\text {impl }}(r)\left(-\boldsymbol{e}_{\phi}\right)=-G M / r^{2} \boldsymbol{e}_{r}
$$

This is the usual expression for centrifugal accelerations in a rotating reference. Note that here, the particle, statonary at a generic point of the ordinary space, in fact is stationary with respect to a reference that is implicitly rotating with respect to the local truly rotating inertial reference. Here $\boldsymbol{g}(r)$ has clearly the nature of a centrifugal acceleration, pointing from everywhere to the gravitational center. $\boldsymbol{g}(r)$ is a spherically symmetric field of centrifugal accelerations toward the gravitational center. A body, effectively hold fixed with respect to the ordinary space coordinates within a gravitational field, necessarily will implicitly be moving along a circular path within the local rotating true IR, round the same overhead axis as this local IR, under a Real upward centripetal force.

Equation (6) is sufficiently precise only for free-fall along short distances. For large distances of free-fall, the elementary linear differential Equation (7) must be solved:

$$
\frac{\mathrm{d} \boldsymbol{v}(r(t))}{\mathrm{d} t}=\boldsymbol{A} \boldsymbol{v}_{0}
$$

where $\boldsymbol{v}$, is the column matrix of the (ordinary) $r$ and the (implicit) $\phi$ velocity components of the velocity with respect to the local HQS:

$$
v(t)=\left(\begin{array}{l}
v_{r}(t) \\
v_{\phi}(t)
\end{array}\right)
$$

In Equation (7), $\boldsymbol{A}$ is the hyperbolic infinitesimal rotation matrix, defined in terms of the rotation rates, given in Equations (5):

$$
\boldsymbol{A}=\left(\begin{array}{cc}
0 & W_{\phi} \mathrm{d} t \\
-W_{r} \mathrm{~d} t & 0
\end{array}\right)=\left(\begin{array}{cc}
0 & W \mathrm{~d} t \\
\frac{1}{u} W \mathrm{~d} t & 0
\end{array}\right)
$$

where $W=\left(G M / r^{3}\right)$ and $u=2 M /(M+m)$ accounts for the asymmetric 
distribution of kinetic energies between the interacting masses $m$ and $M$.

Dividing both sides of Equation (7) by $\boldsymbol{v}_{0}$ and multiplying them by $\mathrm{d} t$ and integrating the left hand side from $\boldsymbol{v}_{0}$ to $\boldsymbol{v}$, develops into:

$$
\log \frac{\boldsymbol{v}(t)}{\boldsymbol{v}_{0}}=\int_{0}^{t} A\left(r\left(t^{\prime}\right)\right) \mathrm{d} t^{\prime}
$$

which can be rewritten in the exponential form as:

$$
\boldsymbol{v}(t)=\boldsymbol{v}_{0} \exp \left[\int_{0}^{t} A\left(r\left(t^{\prime}\right)\right) \mathrm{d} t^{\prime}\right]
$$

Expanding the exponential in series and adding up the terms of the series from $n=0$ to $n=\infty$ results in:

$$
\begin{aligned}
\boldsymbol{v}(t) & =\sum_{n=0}^{\infty} \frac{1}{n !}\left(\begin{array}{cc}
0 & \Theta(t) \\
\frac{\Theta(t)}{u} & 0
\end{array}\right)^{n}\left(\begin{array}{l}
v_{r}(0) \\
v_{\phi}(0)
\end{array}\right) \\
& =\left(\begin{array}{cc}
\cosh \left(\frac{\Theta(t)}{\sqrt{u}}\right) & \sqrt{u} \sinh \left(\frac{\Theta(t)}{\sqrt{u}}\right) \\
\frac{1}{\sqrt{u}} \sinh \left(\frac{\Theta(t)}{\sqrt{u}}\right) & \cosh \left(\frac{\Theta(t)}{\sqrt{u}}\right)
\end{array}\right) \times\left(\begin{array}{l}
v_{r}(0) \\
v_{\phi}(0)
\end{array}\right)
\end{aligned}
$$

The value of $\Theta(t)$ can be computed by integration:

$$
\begin{aligned}
\Theta(t) & =\int_{0}^{t} W\left[r\left(t^{\prime}\right)\right] \mathrm{d} t^{\prime} \\
& =\int_{r_{0} \mathrm{CM}}^{r^{\mathrm{CM}}}\left[\frac{G M}{\left(r^{\mathrm{CM}}+R^{\mathrm{CM}}\right)^{3}}\right]^{1 / 2} \frac{\mathrm{d} r^{\mathrm{CM}}}{\dot{r}^{\mathrm{CM}}} \\
& =-\sqrt{u} \cosh ^{-1}\left[\frac{r_{0}^{\mathrm{CM}}}{r^{\mathrm{CM}}(t)}\right]^{1 / 2}
\end{aligned}
$$

Inversion of the final term of Equation (13) results in expressions for the hyperbolic cosines and sines:

$$
\begin{gathered}
\cosh \left(\frac{\Theta(t)}{\sqrt{u}}\right)=\sqrt{\frac{r_{0}^{\mathrm{CM}}}{r^{\mathrm{CM}}}}=\sqrt{\frac{r_{0}}{r}} \\
\sinh \left(\frac{\Theta(t)}{\sqrt{u}}\right)=\sqrt{\frac{r_{0}^{\mathrm{CM}}-r^{\mathrm{CM}}}{r^{\mathrm{CM}}}}=\sqrt{\frac{r_{0}-r}{r}}
\end{gathered}
$$

where the last equality is obvious. Using this result and noting that $v_{\phi}^{\mathrm{CM}}=v_{\phi}$, Equation (12) becomes:

$$
\left(\begin{array}{c}
v_{r}(t) \\
-v_{\phi}(t)
\end{array}\right)=\left(\begin{array}{l}
\sqrt{r_{0} / r} \sqrt{u\left(r_{0}-r\right) / r} \\
\sqrt{\left(r_{0}-r\right) / u r} \sqrt{r_{0} / r}
\end{array}\right) \times\left(\begin{array}{c}
v_{r}(0) \\
-v_{\phi}(0)
\end{array}\right)
$$

For free fall of $m$ in the field of $M(m \ll M)$, on from $r_{0}$ and initial rest, where $v_{r}(t=0)=0$ and $\boldsymbol{v}_{\phi}(0)=\boldsymbol{V}_{\text {impl }}\left(r_{0}\right)=-\left(G M / r_{0}\right)^{1 / 2} \boldsymbol{e}_{\phi}$, the final solution of Equation (7) is: 


$$
\begin{gathered}
v_{r}(t)=\left[2\left(\frac{G M}{r(t)}-\frac{G M}{r_{0}}\right)\right]^{1 / 2} \\
-v_{\phi}(t)=-V_{\text {impl }}\left(r_{0}\right)\left[\frac{r_{0}}{r}\right]^{1 / 2}=-\left[\frac{G M}{r(t)}\right]^{1 / 2}
\end{gathered}
$$

Equation (16a) is just the well known expression for the observed vertical free-fall on from rest at $r_{0}$, directly showing that the kinetic energy is equal to the variation of the potential energy. Equation (16b) is just the implicit (imaginary) velocity as a function of the radial position $r$. This shows that the refraction rate of the radial velocity component just compensates for the increase of the velocity field as a function of the decrease of the radial coordinate. This assures that free-fall of the particle, on from rest, goes along a vertical (radial) path and hence assures conservation of the angular momentum about the gravitational center. Please observe that, for free-fall on from infinity $\left(r_{0}=\infty\right)$, the vertical velocity $v_{r}(r(t))$ is exactly $\sqrt{2}$ times larger than the $\phi$ velocity $v_{\text {phi }}(r(t))$, exactly as observed, accomplishing the Virial theorem.

\section{Symmetry of the Gravitational Dynamics with Orbital Motions}

Consider free-fall experiments at the equatorial region of a rotating planet like earth. The effective velocity $\boldsymbol{v}_{\text {eff }}$ of a particle, initially stationary near to the surface of a planet, rotating with an angular velocity $\omega$ in the same sense or the opposite sense round the same axis as the Keplerian velocity field, will be given by:

$$
\boldsymbol{v}_{\text {eff }}(\theta)=\boldsymbol{V}_{\text {impl }}(R)+\boldsymbol{v}_{\text {rot }}(\theta)=-\left[(G M / R)^{1 / 2} \mp \omega R \sin \theta\right] \boldsymbol{e}_{\phi}
$$

where $v_{\text {rot }}(\theta)$ is the ordinary velocity, due to the planet's rotation that depends on the latitude via $\sin \theta$. The upper and lower signs are respectively for direct and or retrograde rotation of the planet with respect to that of the local HQS.

However, rotation of the planet in the same sense as the Keplerian velocity field of the HQS, gives rise to a trigonometric rotation rate of the effective velocity $\boldsymbol{v}_{\text {eff }}$ of the particle, due to the planets rotation that adds up to the refraction rate, given by Equation (5b). However, for retrograde rotation, it subtracts, so that the effective rotation rate of the effective velocity vector is:

$$
\boldsymbol{\omega}_{\text {eff }}(\theta)=\frac{1}{R}\left[V_{\text {impl }} \pm\left(v_{\text {rot }}\right)\right] \boldsymbol{e}_{\theta}=\left[\left(G M / R^{3}\right)^{1 / 2} \pm \omega \sin \theta\right] \boldsymbol{e}_{\theta}
$$

where the same convention for the upper and the lower signs as in Equation (17) is used.

Considering the effective velocity (Equation (17)) and the effective rotation rate (Equation (18)), the effective gravitational acceleration on the planet's surface is: 


$$
\boldsymbol{g}_{\text {eff }}(\theta)=\boldsymbol{\omega}_{\text {eff }} \times \boldsymbol{v}_{\text {eff }}=-\left[G M / R^{2}-\omega^{2} R \sin ^{2} \theta\right] \boldsymbol{e}_{r}
$$

The first term in the right hand side of Equation (19) describes the gravitational acceleration toward the gravitational center of the planet in the static situation (see Equation (6)), while the second term is an outward centrifugal term. Equation (19) shows that the effective gravitational acceleration $\boldsymbol{g}_{\text {eff }}(\theta)$ on the rotating planet's surface is perfectly symmetric for direct or retrograde rotation of the planet and thus also for direct or retrograde orbital motion of the particle.

For strictly circular polar orbits with radius $r>R, \quad v_{\text {eff }}$ has velocity components along $-\phi$ as well as along $\theta$. The velocity along $-\phi$ is:

$$
\boldsymbol{v}_{\phi}=\boldsymbol{V}_{\text {imp }}(r)=-[G M / r]^{1 / 2} \boldsymbol{e}_{\phi}
$$

Along theta the velocity is:

$$
\boldsymbol{v}_{\theta}= \pm[G M / r]^{1 / 2} \boldsymbol{e}_{\theta}
$$

While $\boldsymbol{v}_{\phi}$ generates the gravitational acceleration $\boldsymbol{g}(r)=-G M / r^{2} \boldsymbol{e}_{r}$, see Equation (6), the $\theta$ velocity component is not affected directly by the HQS-dynamics, because the velocity field Equation (4) has no velocity component along theta. However, the rotation rate of the $\phi$ velocity component plays the role of a centripetal acceleration toward the gravitational center that bents the $\theta$ velocity toward the earth's surface. The effective gravitational acceleration for polar orbits is:

$$
\boldsymbol{g}_{\text {eff }}(r)=-\left[G M / r^{2}-\left(v_{\theta}^{2}\right) / r\right] \boldsymbol{e}_{r}
$$

where again the first term in the right hand side is the acceleration toward the gravitational center (please see Equation (6)), while the second term is the corresponding usual upward centrifugal effect.

The results, expressed by Equations (19) and (22), show that the effects of the HQS dynamics and the effects of the ordinary motion within the gravitational field are completely independent. They are orthogonal. While the Keplerian velocity field of the HQS (Equation (4)) simulates a central field of fictitious Newtonian gravitational forces, the ordinary orbital motions generate the centrifugal effects, exactly as conceived in Newtonian gravity. This shows that treating the motions within a spherically symmetric gravitational field as motions in a hypothetical inertial reference, extending over the whole region, under a hypothetical central field of fictitious gravitational forces although not corresponding to reality, gives closely the observed gravitational dynamics. This explains why the Newtonian gravitational theory, although based in the fictitious gravitational forces, works so well.

It seems judicious to assume that a Keplerian velocity field of the HQS is circulating round every astronomical body throughout the universe, creating the respective gravitational field. Refs. [3] [4] [5] [6] give details about the HQS dynamics gravitational mechanism, showing that it naturally and accurately 
creates the observed gravitational dynamics on earth and in the solar system. These references also demonstrate that the non-Keplerian velocity field of the HQS, generated by the galaxies, accurately creates even with details the observed non-Keplerian rotation of galaxies, without the need of dark matter.

If earth is very closely stationary with respect to the local HQS, the high velocities of light, of atoms and of elementary particles in the relativistic experiments within the earth-based laboratories, are almost equally high velocity with respect to the local HQS. Within their sensitivity, these relativistic experiments can in no way differentiate between the very similar predictions of the TR and of the theory of the HQS-dynamics. From the HQS-dynamics point of view, the interpretation of all these relativistic experiments on earth, by the usual assumption that the earth-based laboratories can be seen as generic references and arbitrarily be stipulated as hypothetically stationary references of the laboratory observer, clearly is false. It is mistaken because, for all practical instances, earth is not hypothetically, however truly very closely stationary with respect to the local HQS, which is the ultimate reference for rest and for motion for matter-energy. Earth in fact is a very specific preferred reference, closely stationary with respect to the local HQS in the Keplerian velocity field creating the solar and the galactic gravitational fields. In conclusion, the results of all these relativistic experiments do not corroborate the theory of relativity; however simply reveal the true kinematical state of the earth-based laboratories with respect to the local HQS. The absence of the gravitational slowing of the GPS clocks by the solar field and the absence of light anisotropy with respect to the orbiting earth are both the obvious signature of the HQS dynamics physical mechanism of gravity in action.

Anyway however, it must be acknowledged and emphasized that, despite the theory of relativity was constructed without the correct view about the nature of empty space, about the meaning of motions and about the nature of the gravitational fields, Einstein's discoveries about time dilation, relativistic mass and relativistic mass-energy are real and were correctly expressed in terms of velocity. They however are not due to relative velocity. The true cause is velocity with respect to the local HQS, with respect to which earth is almost truly stationary. This however in no way will say that measurements, made outside the environment of earth, too will match the predictions of the theory of relativity.

The Keplerian velocity field of the HQS round earth, in the sense of the Moon's orbital motion (toward the East), achieves $7.91 \mathrm{~km} / \mathrm{sec}$ on surface. The anisotropy effects on light by this velocity is extremely small, only about $10^{-10}$. Despite this, the most sensitive Michelson light anisotropy experiments [18] have detected a nearly West-East light anisotropy of about $8 \mathrm{~km} / \mathrm{sec}$, constant the whole day and the whole year exactly as predicted here. However, recently the one-way velocity of electromagnetic signals (light) was precisely measured in both senses with the help of the atomic clocks, tightly synchronized to within 0.1 
ns in the twin satellites of the GRACE project [21], moving round earth along the same polar orbit and with negligible relative velocity, forming together a well-defined inertial reference in North-South motion. The exchange of electromagnetic signals between these satellites, in both senses, showed a clear anisotropy of the velocity of the signals (light), of nearly $(8 \mathrm{~km} / \mathrm{sec})$ backward to the orbital velocity of these satellites. As the velocity field Equation (4) has no component along the North-South direction, this is exactly the anisotropy predicted by the present HQS-dynamics. This result definitively breaks the postulate of the intrinsic constancy and isotropy of light. Please see Chapter 3 of Ref. [3] for the full details.

\section{Final Comments and Conclusions}

Although conceptually the present approach represents a radical dump of the assumptions of the theory of relativity about the nature of space, the meaning of motions and the nature of the gravitational fields, the difference in the predictions of the two approaches in all of the practical instances on earth is so small that only the recent highly sensitive experimental techniques, with the help of the tightly synchronized atomic clocks in orbit are able to put them in evidence.

Einstein's discoveries about the effects of motion on the clock rate, on mass-energy etc. are of the highest importance. Actually these effects underlie many important frontiers of scientific progress and technological achievements. However, the origin of the inertial mass and of the inertial behavior of the elementary particles remained a mystery until the Higgs theory. The Higgs theory has provided by the first time a scientifically sound explanation for the origin of the inertial mass and the inertial behavior of the elementary particles. According to this theory, Einstein's conclusion that empty space contains nothing that can be a reference for motions was a grave misstep that has lead to the wrong interpretation of the null results of the Michelson light anisotropy experiments. These experimental observations in fact demonstrate that the Higgs Quantum Space (HQS), giving mass to the elementary particles and governing the inertial motion of matter-energy, is moving round the sun according to a Keplerian velocity field, consistent with the planetary motions. This Keplerian velocity field of the HQS is the quintessence of the gravitational fields, in which earth is almost truly stationary with respect to the local HQS, which directly explains the null results of the light anisotropy experiments. These null results together with the absence of the gravitational slowing of the GPS clocks by the solar field are the obvious signature of the physical mechanism of gravity in action that Michelson and Einstein have missed.

\section{References}

[1] Laue, M.V. (1955) Annalen der Physik, 38, 777.

[2] Lorentz, H.A., Einstein, A., Minkowski, H. and Weyl, H. (1923) The Principle of 
Relativity. Dover Publications, New York.

[3] Schaf, J. (2017) The True Origin of the Gravitational Dynamics. Scientific Research Publishing, Inc., Wuhan.

[4] Schaf, J. (2015) Universal Journal of Physics and Application, 9, 141.

[5] Schaf, J. (2014) Recent Progress in Space Technology, 4, 44.

[6] Schaf, J. (2014) Journal of Modern Physics, 5, Article ID: 45323. https://doi.org/10.4236/jmp.2014.56053

[7] Ginzburg, V.L. and Landau, L.D. (1950) Journal of Experimental and Theoretical Physics, 20, 1064-1082.

[8] Higgs, P.W. (1964) Physical Review Letters, 13, 508. https://doi.org/10.1103/PhysRevLett.13.508

[9] Englert, F. and Brout, R. (1964) Physical Review Letters, 13, 321. https://doi.org/10.1103/PhysRevLett.13.321

[10] Kibble, T.W.B. (2009) Scholarpedia, 4, 8741. https://doi.org/10.4249/scholarpedia.8741

[11] Carrol, S.M. (2000) The Cosmological Constant.

[12] Sola, J. (2013) Cosmological Constant and Vacuum Energy: Old and New Ideas.

[13] Anderson, P.W. (1963) Physical Review, 130, 439. https://doi.org/10.1103/PhysRev.130.439

[14] Meissner, W. and Ochsenfeld, R. (1933) Naturwissenschaften, 21, 787-788. https://doi.org/10.1007/BF01504252

[15] Hatch, R.R. (2004) GPS Solutions, 8, 67-73. https://doi.org/10.1007/s10291-004-0092-8

[16] Hatch, R.R. (2004) Foundations of Physics, 34, 1725-1739. https://doi.org/10.1007/s10701-004-1313-2

[17] Ives, H.E. and Stilwell, G.R. (1938) Journal of the Optical Society of America, 28, 215-226. https://doi.org/10.1364/JOSA.28.000215

[18] Miller, D.C. (1933) Review of Modern Physics, 5, 203-242. https://doi.org/10.1103/RevModPhys.5.203

[19] Bailey, H., Borer, K., Combley, F., Drumm, H. and Krienen, F. (1977) Nature, 268, 301-305. https://doi.org/10.1038/268301a0

[20] Ashby, N. (1996) Mercury, 25, 23-27.

[21] Hatch, R.R. (2007) Physics Essays, 20, 83. 